\title{
A STUDY ON THE FLORA ALONG ISMAILIA CANAL
}

(Received:6.9.2012)

\author{
By \\ A. A. Mohamed and S. A. Azer \\ Flora and Phytotaxonomy Researches Department, Horticultural Research \\ Institute, Agricultural Research Center, Dokki, Giza, Egypt
}

\begin{abstract}
A study on the flora along Ismailia canal recorded the presence of 110 species belonging to 94 genera and 34 Angiospermae families. Gramineae was represented by 24 species $(21.8 \%)$, each of Compositae, Leguminosae and Cruciferae were represented by 15 (13.6\%), 8 (7.3\%) and 7 (6.4\%) species, respectively. Five species were recorded from both Convolvulaceae and Solanaceae, while Aizoaceae, Chenopodiaceae and Polygonaceae were represented by 4 species. Three species from both Cyperaceae and Euphorbiaceae; and 2 species from each of Amaranthaceae, Malvaceae, Plantaginaceae, Salicaceae and Umbelliferae. Moreover, 18 families were represented by only 1 species. Annual species in the studied area were 73 species $(66.4 \%$ ); while perennial ones were 32 species $(29.1 \%)$. There were 3 trees $(2.7 \%)$ and 2 shrubs (1.8\%). An endemic species; Trigonella occulta which is considered as a new record to the area of the study was collected. Three species of naturalized flora are recorded which are Aptenia cordifolia, Lycopersicum esculentus and Tricticum aestivum, where there is one parasitic species: Cuscuta campestris. Six hundred and thirty specimens were collected from the studied area and prepared as herbarium sheets.
\end{abstract}

Key words: Flora, floristic category, Ismailia Canal.

\section{INTRODUCTION}

Ismailia canal is the only canal for water supply in the distinct area between Cairo and Ismailia. The Ismailia canal was constructed in 1862 by virtue of two agreements between the Egyptian government and the Suez Canal Company for creating a navigable waterway between the Nile and the Suez Canal. Today its water is only used for irrigation and to provide drinking water for towns along its course. The canal has its inlet from the Nile at Cairo and runs directly to the east to Ismailia town passing the governorates of Cairo, Kalioubeya, Sharkeya and Ismailia. At Ismailia it bifurcates into two arms: one to the north to supply water to the town of Port Said and the second directed toward south to Suez town. A short part without current directly connects the canal to the Suez Canal (Stahl \& Ramadan, 2008). This study is concerning the part from Cairo to Ismailia which extends for 128 $\mathrm{km}$ long and its width is about $30-70 \mathrm{~m}$. A big part leads through sand areas. During the last two millennia, the number of weeds recorded in the flora of Egypt increased to 470 species which constitute the current weed assemblages of Egypt and represent about $22.5 \%$ of the total number of the flowering plants in the Flora of Egypt (Boulos 1995). Many studies were conducted on the weed flora of the Nile islands and irrigation canals. Mashaly and El-Ameir (2007) recorded 70 species through a study on hydrophytic vegetation in the irrigation and drainage canal system of the River Nile in the Delta region. Mohamed and Hassan (1998) conducted a study on the plant life in the Nile islands of Minia governorate. The study dealt with 43 islands stretching on a distance of about $125 \mathrm{~km}$ long. The recorded weed flora of these islands comprised 95 species, including 36 water and canal - bank species (ruderal) as well as 59 (agrestal) species. Shaheen (2002 a \& b) gave an account on some aspects of biodiversity of the weed flora in the farmland of different phytogeographical regions of Aswan, including those of the Nile land (Nile Valley and Nubian Nile Valley). Shaheen et al. (2004) recorded 206 species representing 23 orders, 51 families and 153 genera in a study of botanical diversity in the flora of some islands in the Egyptian Nubia. The loss of biodiversity may take many forms but at its most fundamental irreversible form, it involves 
the extinction of the species. In contrast, the maintenance of variable population of species or identifiable population, however, began to link these basically dissimilar approaches. Few accounts dealt with different aspects of diversity in the weed flora of Egypt (El Hadidi and Hosni, 2000; El Hadidi et al. 1996). This study aimed to make a survey for the flora along Ismailia canal (aquatic plants, helophyte and canal bank weeds) in addition to the seasonally weeds which grow in the narrow strip of cultivated fields along the canal. The specimens collected will be identified and prepared in a form of herbarium sheets.

\section{MATERIALS AND METHODS}

The study was conducted along the region of Ismailia canal from Cairo to Ismailia. Several visits were performed to the area during the period from March 2011 to April 2012. Eight sites (S1S8) were selected according to species diversity along Ismailia canal (Fig. 1). About 630 specimens were collected and prepared as herbarium sheets. These herbarium sheets were preserved in the herbarium of Flora and Phytotaxonomy Researches Department (CIAM), Dokki, Giza, Egypt and arranged according to Engler's system. The specimens were identified according to Täckholm 1974, and Boulos, 1999, 2000, 2002, and 2005. The species recorded were arranged alphabetically within their families. For each species original publication, reference, habitat and life form, floristic category (Raunkiaer 1934) and local names were cited. A map for the studied area is shown in Fig. (1).

\section{RESULTS}

The following is a detailed list according to families of the species mentioned in Table (1) together with their habitat types, life forms, floristic categories and local names.

\subsection{Aizoaceae}

3.1.1. Aizoon canariense L., Sp. Pl., ed. 1, 488 (1753); Boulos 1999, p. 46.

Habitat and life form: Sandy and stony soils. Therophyte.

Floristic category: Palaeotropical.

Local names: Hadaq, Hodaq, Samh, Semeh, Koshed el-belaad.

3.1.2. Aptenia cordifolia (L. f.) N. E. Br., Jour. Bot. 66: 139 (1928).

Habitat and life form: Sandy and stony soils. Chamaephyte.

Floristic category: Cultivated.

Local names: Sabbar israeili.

3.1.3. Trianthema portulacastrum L., Sp. Pl., ed.
1, 223 (1753); Boulos 1999, p. 48.

Habitat and life form: Summer weed in cultivations and waste places. Therophyte.

Floristic category: Palaeotropical.

Local name: Riglh efrangi.

3.1.4. Mesembryanthmum nodiflorum L., Sp. Pl., 1, 481 (1753); Boulos 1999, p.45.

Habitat and life form: Sandy soils and edges of salt marshes. Therophyte.

Floristic category: Med Region.

Local names: Ghasool, Tarteer, Bizz el-kalba, Samh, Semeh.

\subsection{Amaranthaceae}

3.2.1. Amaranthus lividus L., Sp. Pl., ed. 1, 990 (1753); Boulos 1999, p135.

Habitat and life form: Summer weed in cultivations, waste places and irrigation canals.

Therophyte. Floristic category: Cosmopolitan.

Local name: Amaranthoan.

3.2.2. Amaranthus viridis L., Sp. Pl., ed. 2, 1405 (1763); Boulos 1999, p. 135.

Habitat and life form: Summer weed in cultivations, waste places and irrigation canals.

Therophyte.

Floristic category: Palaeotropical.

Local names: Kabshoo-lingah.

\subsection{Asclepiadaceae}

3.3.1. Cynanchum acutum L., Sp. Pl., ed. 1: 212 (1753); Boulos 2000, p. 220.

Habitat and life form: Nile and canal banks, waste moist ground. Hemi-cryptophyte.

Floristic category: Med, Ir-Tur and Sah-Sind Regions.

Local names: $\square$ Ollaiq, $\square$ Olleiq, Moddeid, Motteit, Meddaad, Libbein, Libbeina.

\subsection{Ceratophyllaceae}

3.4.1. Ceratophyllum demersum L., Sp. Pl., ed. 1, 992 (1753); Boulos 1999, p.153.

Habitat and life form: Shallow and deep waters. Hydrophyte.

Floristic category: Cosmopolitan.

Local names: Horeish, Bisht el-qrid, Nakshoosh el-hoot, Abela.

\subsection{Chenopodiaceae}

3.5.1. Atriplex lindleyi Moq. subsp. inflata (F. Muell.) P. G. Wilson, Fl. Australia 4: 323 (1984);

Boulos 1999, p. 99.

Habitat and life form: Sandy soils, waste ground. Therophyte

Floristic category: Pantropical.

Local names: Qataf, Qatf.

3.5.2. Beta vulgaris L., Sp. Pl., ed. 1, 222 (1735); subsp maritima (L.) Arcang.,

Comp. Fl. Ital. 593 (1882); Boulos 1999, p. 94.

Habitat and life form: Weed in winter cultivations 
and along roads sides. Therophyte.

Floristic category: Cosmopolitan.

Local names: Dirs el-kalb, Salq, Silq, Figlbou-leil. 3.5.3. Chenopodium album L., Sp. Pl., ed. 1, 218 (1753); Boulos 1999, p.96.

Habitat and life form: Winter weed in fields and along irrigation canals. Therophyte

Floristic category: Cosmopolitan.

Local names: Rokab el - gamal, Fiss el- kelb ( kilaab ).

3.5.4. Chenopodium murale L., Sp. Pl., ed. 1, 219 (1753); Boulos 1999, p. 96.

Habitat and life form: Weed. Therophyte.

Floristic category: Cosmopolitan.

Local names: Lissan el-teir (el-thor), Qeihaniya, Abu 'efin, Fiss el- kelb (kilaab) Sontaar, Fatteh, Shagaret el-motteina, Fosseish, Sakaraan

\subsection{Compositae}

3.6.1. Ethulia conyzoides L. f., Decas Prima 1, t. 1 (1762) subsp. Conyzoides: Boulos 2002, p. 183.

Habitat and life form: Nile and canal banks. Therophyte.

Floristic category: Palaeotropical.

Local name: Hasheesh el-faras.

3.6.2. Bidens pilosa L., Sp. Pl., ed. 1,832 (1753);

Boulos 2002, p.234.

Habitat and life form: Winter weed on canal banks and ditches. Therophyte.

Floristic category: Pantropical.

Local names: Ebret el-Agooz.

3.6.3. Cichorium endivia L., Sp. Pl., ed. 1, 813 (1753) subsp. divaricatum (Schousb.) P. D. Sell, Bot. J. Linn. Soc. 71: 240 (1976); Boulos 2002, p. 270.

Habitat and life form: Weed of cultivation and moist ground. Therophyte.

Floristic category: Med and Ir-Tur Regions.

Local names: Shikoria, Maqd, Moqd, Sirees, Sablangaaro.

3.6.4. Conyza bonariensis (L.) Cornquist, Bull. Torrey Bot. Club 70: 362 (1943); Boulos 2002; p.192.

Habitat and life form: On Nile and canal banks and in waste places. Therophyte.

Floristic category: Med Region.

Local names: Qessaniya, Howeimda, Billeikh, Belleikh.

3.6.5. Eclipta prostrata (L.) L., Mant. Alt. 286 (1771); Boulos 2002, p. 230.

Habitat and life form: Along Nile and canal banks and in waste places. Therophyte.

Floristic category: Pantropical.

Local names: Sa'da, Soweid, Sawed, Soweid, Swed, Hasheesh el-faras.

3.6.6. Pseudognaphalium luteoalbum (L.) Hilliard
\& B. L. Burtt, Bot. J. Linn. Soc. 82: 206 (1981); Boulos 2002, p. 207.

Habitat and life form: Weed of canal banks, moist ground near springs. Therophyte.

Floristic category: Cosmopolitan.

Local names: Saboon 'afreet, Ghobbeira, Ghobbairaa, Ra'raa' ayyoob

3.6.7. Launaea nudicaulis (L.) Hook. f., Fl. Brit. Ind. 3:416 (1881); Boulos 2002, p. 291.

Habitat and life form: Sandy and alluvial soils, desert plains and wadis, edges of cultivation. Chamaephyte.

Floristic category: Sah - Sind, Med and IR - Tur Regions.

Local names: Ribaay eilaab, Howa, Moraar, Howthaan, Lesseiq, Lisseq, Lossaaq

3.6.8. Pluchea dioscoridis (L.) DC., Prodr. 5: 450 (1836); Boulos 2002, p. 189.

Habitat and life form: Moist ground and canal banks. Chamaephyte.

Floristic category: Sah-Sind, IR-Tur and Med Regions.

Local names: Barnoof.

3.6.9. Pulicaria undulata (L.) C.A.Mey, Verz. Pfl. Casp. Meer. 79 (1831); Boulos 2002, p. 221.

Habitat and life from: desertic plant. Chamaephyte.

Floristic category: Sud - Zamb and Sah - Sind Regions.

Local names: Kootkaat, Rabool, Rabl, Rabd, Ghobbayra, Shaay gabali.

3.6.10. Reichardia tingitana (L.) Roth, Bot. Abh. 35 (1787); Boulos 2002, p. 299.

Habitat and life form: Desert wadis, sandy and alluvial plains, edges of cultivation. Therophyte.

Floristic category: Med and Sah-Sind Regions.

Local names: Galawein, Galaweel, Galaaweel, Nooqd, Libbein, Libbeina, Moraar, Moreir, Meroor, Morayre, Hawa, Howa Howei kelaab, Dordaa, 'Adeed, Shideed

3.6.11. Senecio aegyptius L., Sp Pl., ed. 1, 867 (1753); Boulos 2002, p.265.

Habitat and life form: Silty Nile Banks and irrigation canals. Therophyte.

Floristic category: Sud - Zamb Region.

Local names: Beysoom, Koos .

3.6.12. Senecio glaucus L., Sp. Pl., ed. 1, 868(1753) subsp. coronopifolius (Maire) C. Alexander, Notes Roy. Bot. Gard. Edinb. 37:412(1979) ; Boulos 2002, p. 263.

Habitat and life form: Winter weed especially in new reclaimed lands. Therophyte.

Floristic category: Sah - Sind, Med and IR - Tur Regions.

Local names: Moroor, Moreyre, Meroor, Sofeera, 
Safeery, Sofra, Sofeir - attan.

3.6.13. Sonchus oleraceus L., Sp. Pl., ed. 1,749 (1753); Boulos 2002, p. 309.

Habitat and life form: Common weed in cultivated lands, along canal banks and roadsides. Therophyte.

Floristic and category: Cosmopolitan.

Local names: Go'odied, Goo'dei, Galawein, Galaaweel, Galaeel, Khass, Khaz, Howa, Kass klab, Khass wez, Shekhasheikh, Shikschaakh.

3.6.14. Symphyotrichum squamatum (Spreng.) Nesom, Phytologia 77: 292 (1994); Boulos 2002 p. 194.

Habitat and life form: Weed of cultivation, roadsides, waste ground, and canal banks. Therophyte.

Floristic category: Pantropical.

Local names: Unknown.

3.6.15. Xanthium strumarium L., Sp. Pl., ed. 1, 987 (1753); Boulos 2002, p. 229.

Habitat and life form: Edges of cultivation and waste ground. Therophyte.

Floristic category: Palaeotropical.

Local names: Shobbeit.

\subsection{Convolvulaceae}

3.7.1. Convolvulus arvensis L., Sp. Pl., ed. 1, 153 (1753); Boulos 2000, p. 249.

Habitat and life form: Common weed in cultivations and canal banks all over the year. Chamaephyte.

Floristic category: Palaeotrpical.

Local names: 'Olleiq, Meddaad, Moddied, Motteit, Shagaret el-'Olleyqa.

3.7.2. Convolvulus lanatus Vahl, Symb. Bot. 1: 16 (1790); Boulos 2000, p. 245.

Habitat and life form: Sandy dunes and desert plains. Chamaephyte.

Floristic category: Sah-Sind and Ir-Tur Regions.

Local names: Bayaad, Rakhaam, Rakhaami, Rokhaam, Breheyman, Rehaaman.

3.7.3. Cuscuta campestris Yunck, Mem. Torret Bot. Club 18: 138 (1932); Boulos 2000, p.245.

Habitat and life form: A stem parasite particularly on Trifolium and Capsicum. Parasite.

Floristic category: Pantropical.

Local names: Hamool.

3.7.4. Ipomoea carnea Jacq., Enum. Pl. Carib. 13 (1760); Boulos 2000, p.258.

Habitat and life form: Along roadside sand on canal banks. Phanerophyte.

Floristic category: Pantropical.

Local names: Sitt el-hosn, Bint el-hosn, Mossys.

3.7.5. Ipomoea cairica (L.) Sweet, Hort. Brit. 287 (1827); Boulos 2000, p.259.
Habitat and life form: Canal banks and edges of cultivations. Chamaephyte.

Floristic category: Cultivated and naturalized.

Local name: Sit el-hosn.

\subsection{Cruciferae}

3.8.1. Brassica tournefortii Gouan, Ill. Observ. Bot. 44, t. 20A (1773); Boulos 1999, p.211.

Habitat and life form: Weed in winter crops especially new reclaimed lands. Therophyte.

Floristic category: Med Region with extension to IR-Tur Region.

Local names: Shilltam, Shirtaam.

3.8.2. Eruca sativa Miller, Gard. Dict. Ed. 8, no.1 (1768); Boulos 1999, p. 214.

Habitat and life form: Weed of cultivation, also escape from cultivation. Therophyte.

Floristic category: Med, Ir-Tur, Sah-Sind and Euro-Sib Regions (cultivated).

Local names: Gargeer, Rawq, Shiltaam, Shirtaam, Gery, Afein, Afeen.

3.8.3. Lepidium sativum L., Sp. Pl., ed. 1: 644 (1753); Boulos 1999, p. 225.

Habitat and life form: Weed in winter crops especially in clover fields, also escape from cultivation. Therophyte.

Floristic category: Med Region (Cultivated).

Local names: Habb el-rashaad, Reshaad, Rashaad, Genab, Melwa.

3.8.4. Matthiola longipetala (Vent) DC., Syst. Nat. 2: 174 (1821); Boulos 1999, p. 201.

Habitat and life form: Sandy and stony desert. Therophyte

Floristic category: Med and Sah-Sind Regions.

Local names: Mantoor.

3.8.5. Raphanus raphanistrum L. Sp. Pl., ed. 1, 669 (1753); Boulos 1999, p. 220.

Habitat and life form: Roadsides and edges of cultivation. Therophyte.

Floristic category: Med Region.

Local names: Figl barri.

3.8.6. Sinapis arvensis L., Sp. Pl., ed. 1:668 (1753); Boulos 1999, p. 213.

Habitat and life form: Winter weed in cultivated fields. Therophyte

Floristic category: Med Region.

Local names: Kabar, Khardal, Qirilla.

3.8.7. Sisymbrium irio L. Sp. Pl., ed. 1, 659 (1753); Boulos 1999, p.186.

Habitat and life form: Common winter weed in fields, gardens, orchard and along irrigation canals. Therophyte.

Floristic category: Cosmopolitan.

Local names: Shillyaat, Saleekh, Esseileih, Figl el-gamal. 


\subsection{Cyperaceae}

3.9.1. Cyperus alopecuroides Rottb., Descr. Pl.Rar. Progr. 20 (1772); Boulos 2005, p. 378.

Habitat and life form: Common weed along Nile and canal banks. Chamaephyte.

Floristic category: Pantropical.

Local names: Samaar helw, Koosh, Serakoan asla, 'Aloob, 'Oloob, 'Aloob es-sultaan.

3.9.2. Cyperus articulatus L., Sp. Pl., ed. 1: 44 (1753); Boulos 2005, p. 380.

Habitat and life form: Nile and canal banks. Chamaephyte.

Floristic category: Palaeotropical.

Local names: Boot, Dees medawar.

3.9.3. Cyperus rotundus L., Sp. Pl., ed. 1, 45 91753); Boulos 2005, p.382.

Habitat and life form: Common weed in fields, Nile and canal banks and moist places. Geophyte.

Floristic category: Pantropical.

Local names: Se'd, Sa'd, Sa'd el-homaar, Zibl elmaa'iz, Magessa.

\subsection{Euphorbiaceae.}

3.10.1. Euphorbia heterophylla L., Sp. Pl., ed. 1, 453 (1753); Boulos 2000, p.54.

Habitat and life form: Common summer weed in cultivations and along irrigation canals.

Therophyte.

Floristic category: Pantropical.

Local names: Sa'da.

3.10.2. Euphorbia peplus L., Sp. Pl., ed. 1, 456 (1753); Boulos 2000, p. 64.

Habitat and life from: Winter weed. Therophyte.

Floristic category: Cosmopolitan.

Local names: Wideina, Ma'laqa, Saboon gheit.

3.10.3. Ricinus communis L., Sp. Pl., ed. 1, 1007 (1753); Boulos 2000, p.44.

Habitat and life form: Common plant on Nile and canal banks cultivated and naturalized. Chamaephyte.

Floristic category: Pantropical.

Local names: Kherwa'a, Kharwa'a.

\subsection{Geraniaceae}

3.11.1. Erodium oxyrhynchum M. Bieb., Fl. Taur.Caucas. 2: 133 (1808); Boulos 2000, p. 4.

Habitat and life form: Sandy plains and desert wadis. Chamaephyte.

Floristic category: Sah-Sind Region.

Local names: Morghaat, Dahma, Dahma-a.

\subsection{Gramineae}

3.12.1. Arundo donax L., Sp. Pl., ed. 1, 81 (1753); Boulos 2005, p. 221.

Habitat and life form: Common reed along Nile and canal banks. Helophyte.

Floristic category: Cultivated.
Local names: Ghaab baladi,Ghaab farisi, Ghaab roomi, Ghaab maksoos, Ghaab makaoos, Boos, Boos hagni, Boos rasheedi, Qasab.

3.12.2. Brachiaria mutica (Forssk.) Stapf in Prain, Fl. Trop. Afr. 9: 526 (1919); Boulos 2005, p. 294.

Habitat and life form: Canal banks and irrigation ditches. Chamaephyte.

Floristic category: Cosmopolitan.

Local names: Moddeid, Medaad, Motteit, Rikebeh, Rokeyb.

3.12.3. Cynodon dactylon (L.) Pers., Syn. Pl., 1: 85 (1805); Boulos 2005, p.280.

Habitat and life form: Common weed in fields, gardens and on Nile and canal banks. Geophyte.

Floristic category: Sah-Sind, IR-Tur and SudZamb Regions.

Local names: Nigeel (baladi), Nisheet, Takabalt, Moddied, Meddaad, Motteit.

3.12.4. Desmostachya bipinnata (L.) Stapf in Dyer, Fl. Cap. 7: 632 (1900); Boulos 2005, p.268.

Habitat and life form: Nile and canal banks, along roadsides and in waste places. Geophyte.

Floristic category: Sah-Sind and Sud-Zamb Regions.

Local names: Halfa, Halfa-a.

3.12.5. Digitaria sanguinalis (L.) Scop., Fl. Carniol., ed. 2, 1: 52 (1772); Boulos 2005, p.310.

Habitat and life form: Weed in fields, gardens, along roadsides and on canal banks. Therophyte.

Floristic category: Palaeotropical.

Local names: Dafira.

3.12.6. Echinochloa colona (L.) Link, Hort. Berol. 2: 209 (1833); Boulos 2005, p.291.

Habitat and life form: Common summer weed. Therophyte.

Floristic category: Pantropical.

Local names: Moddeid, Motteit, Abu rokba, Zafra, Beshaft, Hasheesh anaareb.

3.12.7. Echinochloa stagnina (Retz.) P. Beauv., Ess. Agrost. 53: 161 (1812); Boulos 2005, p. 291.

Habitat and life form: Pest in Nile and irrigation canals. Geophyte.

Floristic category: Palaeotropical.

Local names: Omshoot, Mosheet, Nissela, Shaneen, Khaneen, Anneesh, Zommoor.

3.12.8. Hordeum murinum L., Sp. Pl., ed. 1, 85(1753) subsp. glaucum (Steud.) Tzvelev, Novosti Sist. Vyssh. Rast. 1971: 67 (1971); Boulos 2005, p. 207.

Habitat and life form: Desert sand and as a weed of cultivation. Therophyte.

Floristic category: Med and IR-Tur Regions.

Local names: Sheer Barri, Sheera. 
3.12.9. Imperata cylindrica (L.) Raeusch, Nomencl. Bot., ed. 3, 10 (1797); Boulos 2005, p. 327.

Habitat and life form: On canal banks, along roadsides and in waste ground. Hemi Cryptophyte.

Floristic category: Pantropical.

Local names: Halfa, Halfa deil el-Qott, Silla, Sillet, Besto', Boo-doweys, Beniesh-Shaam, Ambarta, Hellein.

3.12.10. Leptochloa fusca (L.) Kunth, Re'vis. Gramin. 1: 91 (1829); Boulos 2005, p. 247.

Habitat and life form: Common weed along Nile and canal banks. Chamaephyte.

Floristic category: Palaeotropical.

Local names: Heesh, Qasaba, Sayfoun.

3.12.11. Lolium multiflorum Lam., Fl. Franc. 3: 621 (1778); Boulos 2005, p. 137.

Habitat and life form: Winter weed. Therophyte.

Floristic category: Med and Euro-Sib Regions.

Local names: Samma, Sammah.

3.12.12. Parapholis marginata Runem., Bot. Notiser 115: 8, t. 3D (1962); Boulos 2005, p. 156.

Habitat and life form: Sandy and stony soils. Therophyte.

Floristic category: Med Region.

Local names: Unknown.

3.12.13. Paspalidium geminatum (Forssk.) Stapf in Prain, Fl. Trop. Afr. 9: 583 (1920); Boulos 2005, p.302.

Habitat and life form: Irrigation ditches, along canals and in swamps. Chamaephyte.

Floristic category: Palaeotropical.

Local names: Sayfoon, Sifoon, Niseela, Qasaba, Qasab, Nigeel dakkar, Abu beid

3.12.14. Pennisetum calndestinum Hochst ex Chivo., Pirotta, Fl. Eritrea 41 (1903); Boulos 2005, p.312.

Habitat and life form: Cultivated for fodder and naturalized. Geophyte.

Floristic category: Palaeotropical.

Local names: Sabat, Sabad, Sabt.

3.12.15. Phalaris minor Retz. Observ. Bot. 3: 8 (1788); Boulos 2005, p. 169.

Habitat and life form: Winter weed. Therophyte.

Floristic category: Med and IR-Tur Regions.

Local names: Sha'eer el-faar, 'Ain el-qott.

3.12.16 Phragmites australis (Cav.) Trin. ex Steud., Nomencl. Bot., ed. 2, 2: 324 (1841); Boulos 2005, p. 223.

Habitat and life form: Moist places and banks of the Nile and irrigation canals. Helophyte.

Floristic category: Pantropical.

Local names: Hagna, Ghaab, Ghaab reehi Boos, Boos khabba, Boos baw, Qasab, Qasab hegaazi,
Heesh, Heesh-maddaad, Aghaaneem, Ighoonaam, Meddaad.

3.12.17. Poa апnиa L., Sp. Pl., ed. 1, 68 (1753); Boulos 2005, p. 146.

Habitat and life form: Weed of fields and gardens. Therophyte.

Floristic category: Cosmopolitan.

Local names: Unknown.

3.12.18. Schismus barbatus (L.) Thell., Bull. Herb. Biossier, se'r. 2, 7: 391 (1756); Boulos 2005, p. 218.

Habitat and life form: Sandy and gravelly soils. Therophyte.

Floristic category: Euro-Sib, IR-Tur and Med Regions.

Local names: Bahma, Bohma, Abumaashi, Safsoof, Zaghab (Zera') el-faar, Abu hereiba, Khafoor.

3.12.19. Setaria verticillata (L.) P. Beauv. Ess. Agrostogr. 51, 178 (1812); Boulos 2005, p. 299.

Habitat and life form: Weed of cultivation. Therophyte.

Floristic category: Cosmopolitan.

Local names: Yadaab, Yadaab gabal, Difra, Qamh el-faar, No'oeim, No'eima, Na'eem, Ne'eym, Barhalimdaab.

3.12.20. Setaria viridis (L.) P. Beauv., Ess. Agrostogr. 51, 171, 178 (1812); Boulos

2005, p.301.

Habitat and life form: Weed in gardens and moist places. Therophyte.

Floristic category: Cosmopolitan.

Local names: Deil (Thayl) el-faar.

3.12.21. Sorghum virgatum (Hack.) Stapf in Prain, Fl. Trop. Afr. 9: 111 (1917); Boulos 2005, p. 332.

Habitat and life form: Weed in cultivations and along Nile and canal banks. Therophyte.

Floristic category: Sud-Zamb Region.

Local names: Hasheesh el-faras, Garawa.

3.12.22. Sporobolus spicatus (Vahl) Kunth. Révis. Gramin. 1: 67 (1829); Boulos 2005, p. 271.

Habitat and life form: Seasonally inundated saline desert sand. Chamaephyte.

Floristic category: Med and Sah-Sind Regions.

Local names: Nigeel shetaani, Samma, Sammah, Sill, Silla, Sillet, Maboos, Albristi, Sabat gebeli, Sabat, Sabt,Sabad, Abu rokba, Sakham, Sakhaam, Nigeel shoaki.

3.12.23. Stipa capensis Thunb., Prodr. Fl. Cap. 1: 19 (1794); Boulos 2005, p. 132.

Habitat and life form: Desert and coastal sands and rocky slopes. Therophyte.

Floristic category: Med and Sah-Sind Regions.

Local names: Sabat, Sabad, Safsoof, Sabal abu elhossein, Abu fakhoor, Dreira. 
3.12.24. Triticum aestivum L., Sp. Pl., ed. 1, 85 (1753); nom. Conserve., Boulos 2005, p.212.

Habitat and life form: Escape from cultivation. Therophyte.

Floristic category: Cultivated.

Local names: Qamh.

\subsection{Labiatae}

3.13.1. Lamium amplexicaule L.,Sp. Pl., ed. 1, 579 (1753); Boulos 2002, p. 24.

Habitat and life form: Weed of cultivations. Therophyte.

Floristic category: Med, Ir-Tur and Euro-Sib Regions.

Local names: Taqiyit el-ghoraab, Fom elsamakah.

\subsection{Leguminosae}

3.14.1. Alhagi graecorum Boiss., Diagn. Pl. Orient. 9: 114 (1849); Boulos 1999, p. 340.

Habitat and life form: Weed in waste places and along canal and Nile banks. Hemi-

cryptophyte.

Floristic category: Palaeotropical.

Local names: 'Aqool, 'Agool, Shoak, Shoak elgamal, Afisoor.

3.14.2. Melilotus indicus (L.) All., Fl. Pedem. 1: 308 (1785); Boulos 1999, p.278.

Habitat and life form: Common winter weed. Therophyte.

Floristic category: Palaeotropical.

Local names: Hesheita, Hisheita, Mosheta , Hendaqooq morr, Handaqooq helw 'Atoor ('Otoor) el- khadm, Qort, Nafla, Nefl.

3.14.3. Sesbania sesban (L.) Merr., Philipp. J. Sci. (Bot.) 7: 235 (1912); Boulos 1999, p.319.

Habitat and life form: Nile and canal banks sometimes weed in cultivations.

Nanophanerophyte.

Floristic category: Sud-Zamb Region.

Local names: Sesebaan, Seisabaan.

3.14.4. Trifolium resupinatum L., Sp. Pl., ed. 1, 771 (1753); Boulos 1999, p. 281.

Habitat and life form: Common winter weed in cultivations especially along irrigation canal banks. Therophyte.

Floristic category: Med and IR - Tur Regions extending into Euro - Sib Region.

Local names: Qort, Goreida, Loeyna, Khondeyshe.

3.14.5.Trigonella glabra Thunb. Pl. Cap: 137 (1800); El - Hadidi \& Fayed 1994, p.265.

Habitat and life form: Weed. Therophyte.

Floristic category: Sud - Zamb Med and Sah Sind Regions.

Local names: 'Eshb ( Oshb) el- malik, Daraqraq, Deraaq, Daraaq, Zeraqraaq.
3.14.6. Trigonella laciniata L., Sp. Pl., ed. 2, 1095 (1763); Boulos 1999, p. 265.

Habitat and life form: Sandy soils. Therophyte.

Floristic category: Sud - Zamb and Sah Sind Regions.

Local names: Deraaq, Daraqraaq.

3.14.7. Trigonella occulta Ser. in DC., Prodr. 2: 185 (1825); Boulos 1999, p. 264.

Habitat and life form: Sandy soils. Therophyte.

Floristic category: Endemic.

Local names: Handaqoaq, Handaqooq.

3.14.8. Trigonella stellata Forssk., Fl. Aegypt.Arab. 140 (1775); Boulos 1999, p. 264.

Habitat and life form: Winter weed in cultivations. Therophyte.

Floristic category: Sud - Zamb Med and Sah Sind Regions.

Local names: Gargas, Shetn el-khaadem, Atoor garm, Hesheita, Hisheita.

\subsection{Malvaceae}

3.15.1. Malva parviflora L., Sp. Pl., ed. 2, 926 (1763); Boulos 2000, p. 94

Habitat and life form: Winter weed in cultivated lands and along Nile and canal banks. Therophyte. Floristic category: Med, Euro - Sib, IR- Tur and Sah - Sind Regions.

Local names: Raqma, Raqmiya, Khobbeiza, Khobaaza.

3.15.2. Sida alba L., Sp. Pl., ed. 2, 960 (1752); Boulos 2000, p.100.

Habitat and life form: Robust summer weed. Chamaephyte.

Floristic category: Pantropical.

Local names: Meloukhiet Eblees.

\subsection{Molluginaceae}

3.16.1. Glinus lotoides L., Sp. Pl., ed. 1, 463 (1753); Boulos 1999, p.41.

Habitat and life form: Weed on silty banks and moist places. Therophyte.

Floristic category: Palaeotropical.

Local names: Ghobbeira, Ghobbairaa, Hasheesh el-'aqrab, Damaseisa, Damsees, Dimsees, Demsees, Moghera.

\subsection{Onagraceae}

3.17.1. Ludwigia stolonifera (Guillemin \& al.) P. H. Raven, in Reinwardtia 6: 390 (1964); Boulos 2000, p. 150.

Habitat and life form: In and along channels. Hemi-cryptophyte.

Floristic category: Sud-Zamb Region.

Local names: Moddeid, Moddaad, Motteit, Looq, Aroos el-bahr, Qateef, Qateefa, Halmos, Kharaawa, Freikaal, Forga $\square$ a, Ferga $\square$ a.

\subsection{Oxalidaceae}

3.18.1. Oxalis corniculata L., Sp. Pl., ed. 1, 435 
(1753). Boulos 1999, p.375.

Habitat and life form: Moist shady places and along irrigatios canal banks, Hemi- cryptophytes. Floristic category: Cosmopolitan.

Local names: Hamd, Hommeid.

\subsection{Palmae}

3.19.1. Phoenix dactylifera L., Sp. Pl., ed. 1, 1188 (1753); Boulos 2005 p. 117.

Habitat and life form: Cultivated every where .Phanerophyte.

Floristic category: Sud - Zamb and Sah - Sind Regions.

Local names: Nakhel el-balah.

\subsection{Plantaginaceae}

3.20.1. Plantago lagopus L., Sp. Pl., ed. 1, 114 (1753); Boulos 2002, p. 116.

Habitat and life form: Weed of irrigated gardens and olive groves, natural winter-wet ground. Therophyte.

Floristic category: Med and Ir-Tur Regions.

Local name: Widna.

3.20.2. Plantago major L., Sp. Pl., ed. 1,112 (1753); Boulos 2002 p. 117.

Habitat and life form: Nile and canal banks,irrigated fields and open moist ground. Therophyte.

Floristic category: Cosmopolitan.

Local names: Lisaan hamad, Waraaq saboon, Masaas, Mesaasa, Lisaan el-kalb.

\subsection{Polygonaceae}

3.21.1. Emex spinosa (L.) Campd., Monogr. Rumex 58, t. 1 (1819); Boulos 1999, p. 24.

Habitat and life form: Winter weed on cultivations and canal banks. Therophyte.

Floristic category: Med and Sah - Sind Regions with extension to IR - Tur Region.

Local names: Drs el-ágooz, Rookbet el-agooz, Shagaret el-agooz, Tablaalast, Gazeesi, Hommeid, Shobeit, Hanzaab, Widna, Figl el-gabl, Losseiq, Lossaaq, Lessaaq.

3.21.2. Persicaria sengalensis (Meisn.) Soja'k, Preslia 46: 155 (1974); Boulos 1999, p.27.

Habitat and life form: Nile and canal banks. Helophyte.

Floristic category: Pantropical.

Local names: Laah, Lisaan el-asfoor, Habaq elbahr Bizz el-kalba, Zilf, Abu zilf.

3.21.3. Rumex dentatus L., Mant. Alt. 226 (1771); Boulos 1999, p. 31.

Habitat and life form: Weed in farmland and moist places. Therophyte.

Floristic category: Med, Euro-Sib and IR-Tur Regions.

Local names: Khilla, Khella, Khilaal, Khillaala, Hommeid, Hommaad.
3.21.4. Rumex vesicarius L. Sp. Pl., ed. 1, 336 (1753); Boulos 1999, p. 34.

Habitat and life form: Rocky and sandy desert wadis. Therophyte.

Floristic category: Med, and Sah-Sind Regions.

Local names: Hambeit, Hanbeit, Hamaad, Hammaad, Hammaad el 'eshb.

\subsection{Pontederiaceae}

3.22.1. Eichhornia crassipes (C. Mart.) Solms, in A. \& C. DC., Monogr. Phan. 4: 527 (1883); Boulos 2005, p. 90.

Habitat and life form: Dangerous water pest in Nile and irrigation canals. Hydrophyte.

Floristic category: Palaeotropical.

Local names: Ward en-nil, Halassandi, Jasint elmayya, Zoqeim et-tani, Baqaaqa.

\subsection{Portulacaceae}

3.23.1. Portulaca oleracea L.,Sp. Pl.,ed. 1, 445 (1753); Boulos 1999, p.49.

Habitat and life form: Weed in cultivations and in waste and muddy places. Therophyte.

Floristic category: Cosmopolitan.

Local names: Rigla, Rigla roomi, Ragl, Rashaad.

\subsection{Potamogetonaceae}

3.24.1. Potamogeton nodosus Poiret, in Lam., Encycl. Suppl. 4: 535 (1817); Boulos 2005, p. 18

Habitat and life form: Submerges plant in Nile water and irrigation canals. Hydrophyte.

Floristic category: Cosmopolitan.

Local names: Reem, Hamool, Waraqa, Sheneen, Zafoon.

\subsection{Primulaceae}

3.25.1. Anagallis arvensis L., Sp. Pl., ed. 1, 148 (1753); Boulos 2000, p. 190.

Habitat and life form: Common winter weed in crops and moist habitat. Therophyte.

Floristic category: Cosmopolitan.

Local names: 'Ebeila (blue forms), 'Ain el-gamel, Saboon gheit, Ommleben.

\subsection{Ranunculaceae}

3.26.1. Ranunculus scleratus L., Sp. Pl., ed. 1: 551 (1753); Boulos 1999, p. 145.

Habitat and life form: In channels and moist places. Therophyte.

Floristic category: Euro-Sib Region with extension to Med and IR-Tur Regions.

Local names: Zaghalanta.

\subsection{Resedaceae}

3.27.1. Oligomeris linifolia (Vahl ex Hornem.) J. F. Macbr., Contr. Gray Herb., ser. 2, 23: 13 (1918); Boulos 1999, p. 237.

Habitat and life form: Sandy and stony deserts and plains. Therophyte.

Floristic category: Cosmopolitan. 
Local names: Danabaan, Denabaan, Khozaama, Khazaama, Khesaama.

\subsection{Salicaceae}

3.28.1. Salix mucronata Thunb., Prodr. Pl. Cap. 6 (1794); Boulos 1999, p.13.

Habitat and life form: Nile and canal banks. Phanerophyte.

Floristic category: Palaeotropical.

Local names: Safsaaf baladi.

3.28.2. Salix tetrasperma Roxb., Pl. Coast Corom. 1: 66, t. 97 (1798); Boulos 1999, p.13.

Habitat and life form: Nile and canal banks. Phanerophyte.

Floristic category: Cultivated.

Local names: Safsaaf afrangi.

\subsection{Solanaceae}

3.29.1. Datura innoxia Mill., Gard. Dict. ed. 8, no. 5 (1768); Boulos 2002, p. 46.

Habitat and life form: waste places. Therophyte.

Floristic category: Pantropical.

Local names: Datoora, Tatoora.

3.29.2. Lycopersicum esculentum Miller, Gard. Dict. ed. 8, no. 2 (1768); Hepper 1998, p. 55.

Habitat and life form: Cultivated vegetable crop. Therophyte.

Floristic category: Cultivated.

Local names: Tamatem, Qoutta.

3.29.3. Nicotiana plumbaginifolia Viv., Elench. Pl. Hort. Bot. 26, t. 5 (1802); Boulos 2002, p. 54.

Habitat and life form: Moist ground and canal banks, naturalized. Therophyte.

Floristic category: Pantropical.

Local names: Unknown.

3.29.4. Solanum nigrum L., Sp. Pl., ed. 1, 186 (1753); Boulos 2002, p. 39.

Habitat and life form: Common weed in fields and waste places. Therophyte.

Floristic category: Cosmopolitan.

Local names: 'Anab ('Enab) ed-deeb, Bandoretdeeb.

3.29.5. Withania somnifera (L.) Dunal in DC., Prodr. 13 (1): 5, 453 (1852); Boulos 2002, p.41.

Habitat and life form: Common weed on Nile and canal banks and in waste places. Chamaephyte.

Floristic category: Sah-Sind and Sud-Zamb Regions.

Local names: Semm el-firaakh, Morgaan, Foqqeish, Sherma, Hemer el-ghoraab, Semm elfaar, Shagaret el-ghoraab, Labbakh, Khasraqoot, 'Igshi el-ghoraab, Sakaraan.

\subsection{Tamaricaceae}

3.30.1. Tamarix nilotica (Ehrenb.) Bunge, Tent. Tamaric, 54 (1852); Boulos 200, p. 127.

Habitat and life form: Wide wadis with sandy silty ground . Nanophanerophyte.
Floristic category: Sah - Sind, Sud - Zamb and IR - Tur Regions.

Local names: Abal, Atl and Athal.

3.31. Tiliaceae

3.31.1. Corchorus olitorius L., Sp. Pl., ed. 1, 529 (1753); Boulos 2000, p.88.

Habitat and life form: Cultivated and weed summer in crops. Therophyte.

Floristic category: Pantropical.

Local names: Melokhiya.

\subsection{Typhaceae}

3.32.1. Typha domingensis (Pers.) Poir. ex Steud., Nomencl. Bot. 860 (1824); Boulos 2005, p. 117.

Habitat and life form: Canals, ditches, springs and edges of lakes. Helophyte.

Floristic category: Pantropical.

Local names: Bordi, Bardi, Birdi, Berdi, Dees, Timeyn.

\subsection{Umbelliferae}

3.33.1. Ammi majus L., Sp. Pl., ed. 1, 243 (1753); Boulos 2000, p. 168.

Habitat and life form: Winter weed in moist waste places. Therophyte.

Floristic category: Cosmopolitan.

Local names: Khilla sheitaani.

3.33.2. Apium leptophyllum (Pers.) F. Muell. ex Benth., Fl. Austral. 3: 372 (1867); Boulos 2000, p. 163.

Habitat and life form: Weed of cultivation, roadsides and canal banks. Therophyte.

Floristic category: Palaeotropical \& Pantropical.

Local names: Unknown.

\subsection{Zygophyllaceae}

3.34.1. Zygophyllum simplex L., Mant. 68 (1767), Boulos 2000, p. 23

Habitat and life form: Sandy soils. Therophyte.

Floristic category: Sah - Sind and IR - Tur Regions.

Local names: Garmal, Qarmal, Goormeil.

\section{DISCUSSION}

The study on the flora along Ismailia canal showed the presence of 110 species belonging to 94 genera and 34 Angiospermae families. Gramineae was represented by 24 species ( 21.8 $\%$ ), each of Compositae, Leguminosae and Cruciferae were represented by 15,8 and 7 species, respectively. Five species were recorded from both of Convolvulaceae and Solanaceae, while Aizoaceae, Chenopodiaceae and Polygonaceae were represented by 4 species. Three species from both Cyperaceae and Euphorbiaceae; and 2 species from each of Amaranthaceae, Malvaceae, Plantaginaceae, Salicaceae and Umbelliferae, while 18 families 
Table (1): Flora species recorded along Ismailia Canal, their families and life cycles.

\begin{tabular}{|c|c|c|}
\hline Species & Family & Life cycle \\
\hline Aizoon canariense L. & Aizoaceae & Annual \\
\hline Alhagi graecorum Boiss. & Leguminosae & Perennial \\
\hline Amaranthus lividus L. & Amaranthaceae & Annual \\
\hline Amaranthus viridis $\mathrm{L}$. & Amaranthaceae & Annual \\
\hline Ammi majus L. & Umbelliferae & Annual \\
\hline Anagallis arvensis L. & Primulaceae & Annual \\
\hline Apium leptophyllum (Pers.) F. Muell. ex Benth. & Umbellifereae & Annual \\
\hline Aptenia cordifolia (L. f.) N. E. Br. & Aizoaceae & Perennial \\
\hline Arundo donax $\mathrm{L}$. & Gramineae & Perennial \\
\hline Atriplex lindleyi Moq. subsp. inflata (F. Muell.) P. G. Wilson & Chenopodiaceae & Annual \\
\hline Beta vulgaris L. & Chenopodiaceae & Annual \\
\hline Bidens pilosa $\mathrm{L}$. & Compositae & Annual \\
\hline Brachiaria mutica (Forssk.) Stapf & Gramineae & Annual \\
\hline Brassica tournefortii Gouan & Cruciferae & Annual \\
\hline Ceratophyllum demersum $\mathrm{L}$. & Ceratophyllaceae & Perennial \\
\hline Chenopodium album L. & Chenopodiaceae & Annual \\
\hline Chenopodium murale L. & Chenopodiaceae & Annual \\
\hline Cichorium endivia L., subsp. divaricatum (Schousb.) P. D. Sell & Compositae & Annual \\
\hline Convolvulus arvensis $\mathrm{L}$. & Convolvulaceae & Perennial \\
\hline Convolvulus lanatus Vahl & Convolvulaceae & Perennial \\
\hline Conyza bonariensis (L.) Cornquist & Compositae & Annual \\
\hline Corchorus olitorius L. & Tiliaceae & Annual \\
\hline Cuscuta campestris Yunck. & Convolvulaceae & Annual \\
\hline Cynanchum acutum $\mathrm{L}$. & Asclepiadaceae & Perennial \\
\hline Cynodon dactylon (L.) Pers. & Gramineae & Perennial \\
\hline Cyperus alopecuroides Rottb. & Cyperaceae & Perennial \\
\hline Cyperus articulatus $\mathrm{L}$. & Cyperaceae & Perennial \\
\hline Cyperus rotundus L. & Cyperaceae & Perennial \\
\hline Datura innoxia Mill. & Solanaceae & Annual \\
\hline Desmostachya bipinnata (L.) Stapf & Gramineae & Perennial \\
\hline Digitaria sanguinalis (L.) Scop. & Gramineae & Annual \\
\hline Echinochloa colona (L.) Link & Gramineae & Annual \\
\hline Echinochloa stagnina (Retz.) P. Beauv. & Gramineae & Perennial \\
\hline Eclipta prostrata (L.) L. & Compositae & Annual \\
\hline Eichhornia crassipes (C. Mart.) Solms & Pontederiaceae & Perennial \\
\hline Emex spinosa (L.) Campd. & Polygonaceae & Annual \\
\hline Erodium oxyrhynchum M. Bieb. & Geraniaceae & Perennial \\
\hline Eruca sativa Miller & Cruciferae & Annual \\
\hline Ethulia conyzoides L. f. & Compositae & Annual \\
\hline Euphorbia heterophylla L. & Euphorbiaceae & Annual \\
\hline Euphorbia peplus L. & Euphorbiaceae & Annual \\
\hline Glinus lotoides L. & Mollunginaceae & Annual \\
\hline Hordeum murinum L. subsp. glaucum (Steud.) Tzvelev & Gramineae & Annual \\
\hline Imperata cylindrica (L.) Raeusch & Gramineae & Perennial \\
\hline Ipomoea cairica $(\mathrm{L}$.$) Sweet$ & Convolvulaceae & Perennial \\
\hline Ipomoea carnea Jacq. & Convolvulaceae & Perennial \\
\hline Lamium amplexicaule L. & Labiatae & Annual \\
\hline Launaea nudicaulis (L.) Hook. f. & Compositae & Perennial \\
\hline Lepidium sativum $\mathrm{L}$. & Cruciferae & Annual \\
\hline Leptochloa fusca (L.) Kunth & Gramineae & Perennial \\
\hline Lolium multiflorum Lam. & Gramineae & Annual \\
\hline Launaea nudicaulis (L.) Hook.f. & Compositae & Perennial \\
\hline Lepidium sativum $L$. & Cruciferae & Annual \\
\hline Leptochloa fusca (L.) Kunth & Gramineae & Perennial \\
\hline Lolium multiflorum Lam. & Gramineae & Annual \\
\hline
\end{tabular}




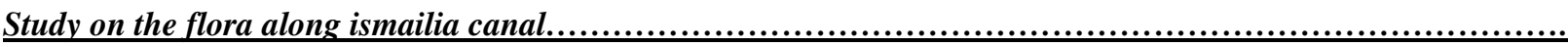

Table (1) Cont.

\begin{tabular}{|c|c|c|}
\hline Species & Family & Life cycle \\
\hline Ludwigia stolonifera (Guillemin \& al.) P. H. Raven & Onagraceae & Perennial \\
\hline Lycopersicum esculentum Miller & Solanaceae & Annual \\
\hline Malva parviflora L. & Malvaceae & Annual \\
\hline Matthiola longipetala (Vent) DC. & Cruciferae & Annual \\
\hline Melilotus indicus (L.) All. & Leguminosae & Annual \\
\hline Mesembryanthmum nodiflorum L. & Aizoaceae & Annual \\
\hline Nicotiana plumbaginifolia Viv. & Solanaceae & Annual \\
\hline Oligomeris linifolia (Vahl ex Hornem.) J. F. Macbr. & Resedaceae & Annual \\
\hline Oxalis corniculata L. & Oxalidaceae & Annual \\
\hline Parapholis marginata Runem. & Gramineae & Annual \\
\hline Paspalidium geminatum (Forssk.) Stapf & Gramineae & Perennial \\
\hline Pennisetum calndestinum Hochst. ex Chivo. & Gramineae & Perennial \\
\hline Persicaria sengalensis (Meisn.) Soja'k & Polygonaceae & Perennial \\
\hline Phalaris minor Retz. & Gramineae & Annual \\
\hline Phoenix dactylifera L. & Palmae & Tree \\
\hline Phragmites australis (Cav.) Trin. ex Steud. & Gramineae & Perennial \\
\hline Plantago lagopus L. & Plantaginaceae & Annual \\
\hline Plantago major L. & Plantaginaceae & Annual \\
\hline Pluchea dioscoridis (L.) DC. & Compositae & Perennial \\
\hline Poa annua L. & Gramineae & Annual \\
\hline Portulaca oleracea L. & Portulacaceae & Annual \\
\hline Potamogeton nodosus Poiret & Potamogetonaceae & Perennial \\
\hline Pulicaria undulata (L.) C.A.Mey & Compositae & Perennial \\
\hline Pseudognaphalium luteoalbum (L.) Hilliard \& B. L. Burtt & Compositae & Annual \\
\hline Ranunculus scleratus L. & Ranunculaceae & Annual \\
\hline Raphanus raphanistrum L. & Cruciferae & Annual \\
\hline Reichardia tingitana (L.) Roth & Compositae & Annual \\
\hline Schismus barbatus (L.) Thell. & Gramineae & Annual \\
\hline Ricinus communis L. & Euphorbiaceae & Perennial \\
\hline Rumex dentatus L. & Polygonaceae & Annual \\
\hline Rumex vesicarius L. & Polygonaceae & Annual \\
\hline Salix mucronata Thunb. & Salicaceae & Tree \\
\hline Salix tetrasperma Roxb. & Salicaceae & Tree \\
\hline Senecio aegyptius L. & Compositae & Annual \\
\hline Senecio glaucus L., subsp. coronopifolius (Maire) C. Alexander & Compositae & Annual \\
\hline Sesbania sesban (L.) Merr. & Leguminosae & Shrub \\
\hline Setaria verticillata (L.) P. Beauv. & Gramineae & Annual \\
\hline Setaria viridis (L.) P. Beauv. & Gramineae & Annual \\
\hline Sida alba L. & Malvaceae & Annual \\
\hline Sinapis arvensis L. & Cruciferae & Annual \\
\hline Sisymbrium irio L. & Cruciferae & Annual \\
\hline Solanum nigrum L. & Solanaceae & Annual \\
\hline Sonchus oleraceus L. & Compositae & Annual \\
\hline Sorghum virgatum (Hack.) Stapf & Gramineae & Annual \\
\hline Sporobolus spicatus (Vahl) Kunth. & Gramineae & Perennial \\
\hline Stipa capensis Thunb. & Gramineae & Annual \\
\hline Symphyotrichum squamatum (Spreng.) Nesom & Compositae & Annual \\
\hline Tamarix nilotica (Ehrenb.) Bunge & Tamaricaceae & Shrub \\
\hline Trianthema portulacastrum L. & Aizoaceae & Annual \\
\hline Trifolium resupinatum L. & Leguminosae & Annual \\
\hline Trigonella glabra Thunb. & Leguminosae & Annual \\
\hline Trigonella laciniata L. & Leguminosae & Annual \\
\hline Trigonella occulta Ser. in DC. & Leguminosae & Annual \\
\hline Trigonella stellata Forssk. & Leguminosae & Annual \\
\hline Triticum aestivum L. & Gramineae & Annual \\
\hline Typha domingensis (Pers.) Poir. ex Steud. & Typhaceae & Perennial \\
\hline Withania somnifera (L.) Dunal & Solanaceae & Perennial \\
\hline Xanthium strumarium L. & Compositae & Annual \\
\hline Zygophyllum simplex L. & Zygophyllaceae & Annual \\
\hline
\end{tabular}




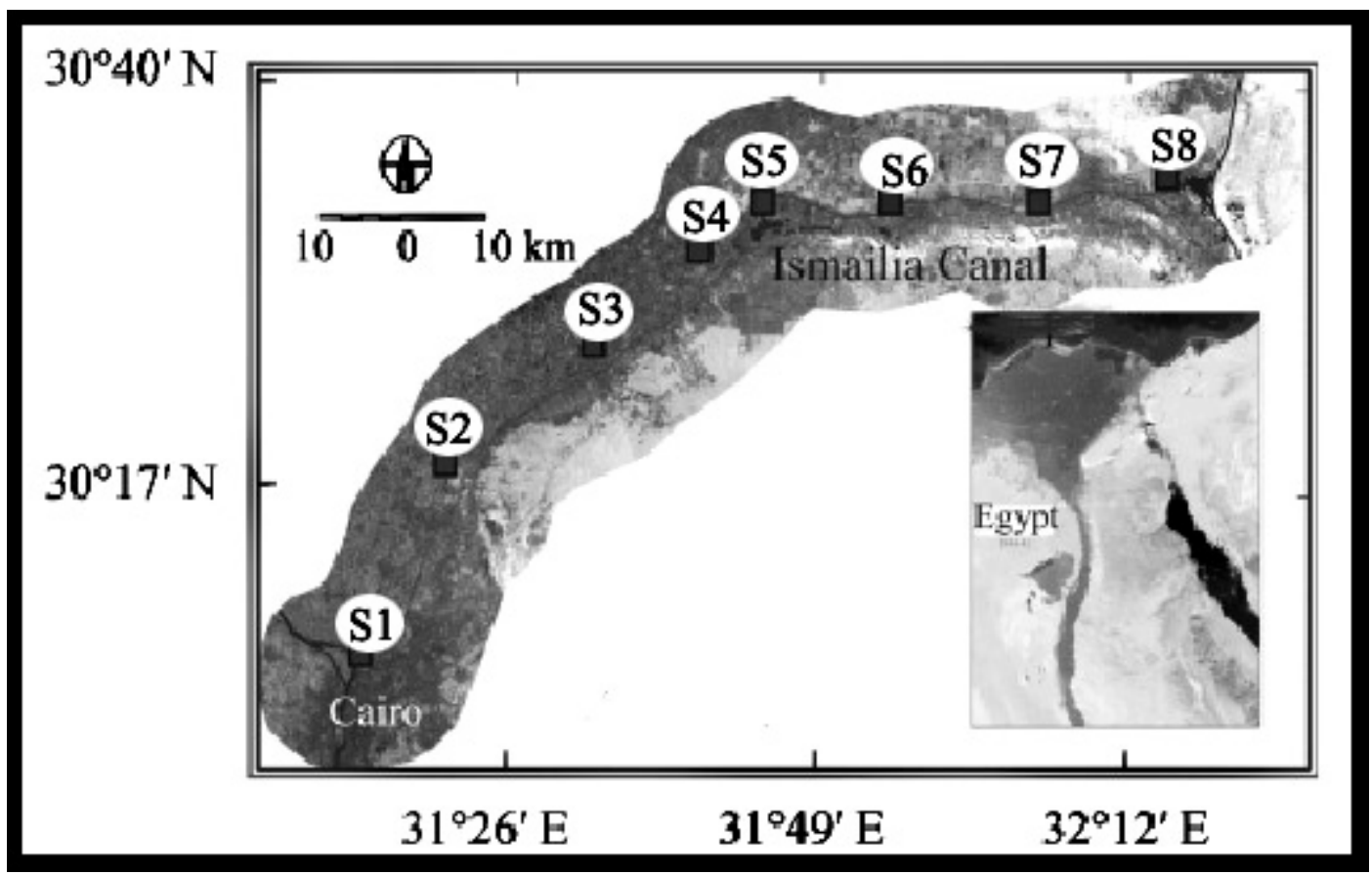

Fig. (1): A map of Ismailia Canal showing the studied area (S1-S8).

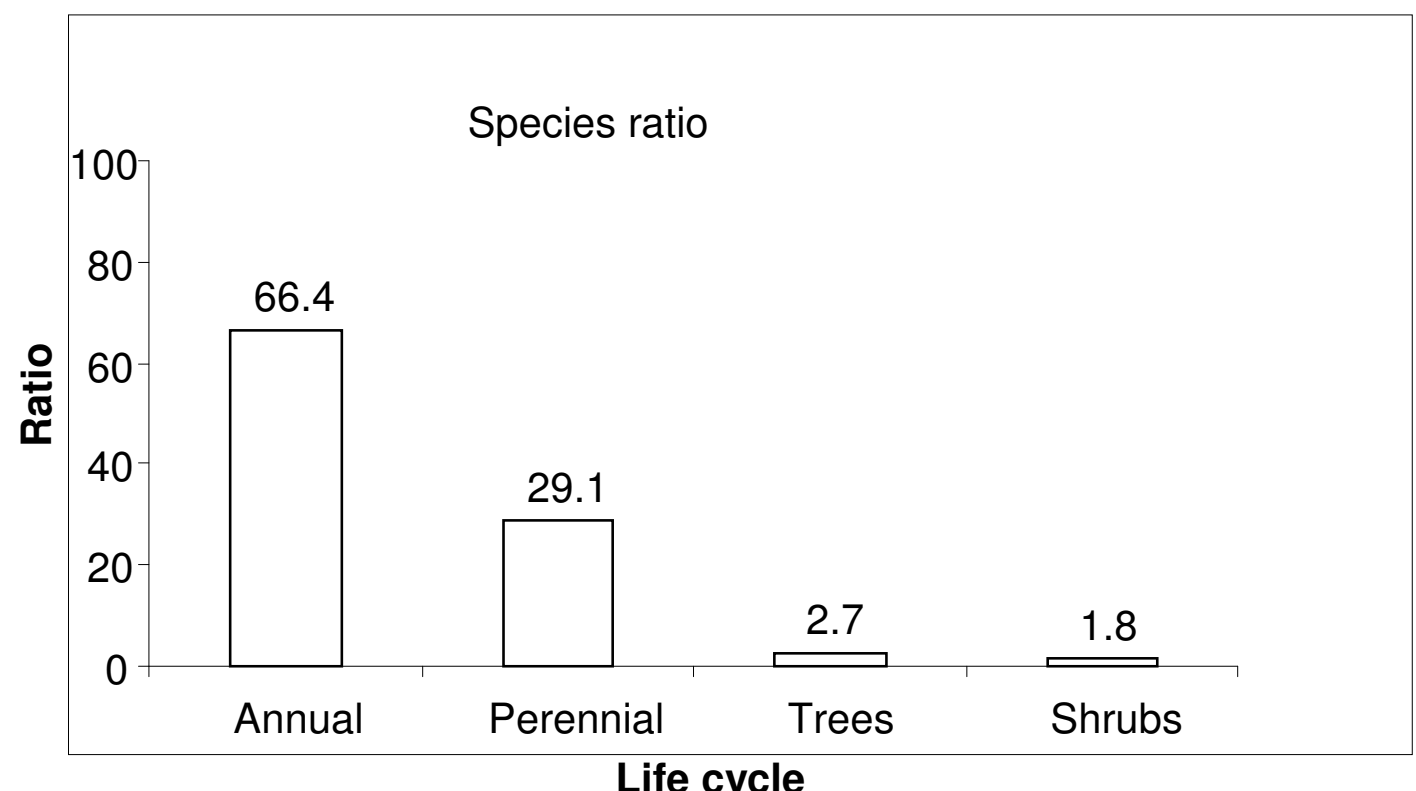

Fig. (2): Spectrum of the life cycle ratios of the studied species along Ismailia canal

were represented only by 1 species. Annual species in the studied area were 73 species $(66.4$ $\%)$; while perennial ones were 32 species $(29.1 \%)$. There were 3 trees $(2.7 \%)$ and 2 shrubs $(1.8 \%)$ Fig (2). An endemic species; Trigonella occulta which is considered as new record to the area of study was collected. There are 3 species recorded as escape from cultivation, Lycopersicum esculentus and Triticum aestivum. Moreover, Cuscuta campestris is the parasitic species recorded from the area of study.

\section{REFERENCES}

Boulos L.(1995). Flora of Egypt; Chek List, AlHadara Puplishing. pp 285.

Boulos L.(1999). Flora of Egypt. vol. I. AlHadara Publishing. Cairo, Egypt.

Boulos L. (2000). Flora of Egypt. vol.II. AlHadara Publishing. Cairo, Egypt.

Boulos L. (2002). Flora of Egypt. vol. III. Al Hadara Publishing. Cairo, Egypt.

Boulos L. (2005). Flora of Egypt. vol. IV. Al Hadara Publishing. Cairo, Egypt. 
El-Hadidi M. N. and Fayed A (1994/95). Materials for excursion flora of Egypt, Cairo University Herbarium. Täeckholmia. 15: 1233.

El Hadidi M. N. and Hosny H. A. ( 2000 ): Flora Aegyptiaca. vol.I, part I, (M. N.El Hadidi ed.). Palm Press, Cairo, Egypt. 187 pp.

El Hadidi M. N., Hosny A. I. and El Husseini N. (1996). Some aspects of biodiversity of weed flora in the farm lands of Egypt in: The biodiversity of African plants. L.J.G. vander Maesen et al., (eds), Kluwer Academic Publishers, Netherlands, 788-794.

Hepper F.N. (1998). Family 159. Solanaceae. Täeckholmia Additional Series, 6: 1-68. Cairo University Herbarium, Egypt.

Mashaly I. A. and El-Ameir Y. A. (2007). Hydrophytic vegetation and drainage canal system of the River Nile in Egypt. World App. Sci. Jour. 2 (1): 49-61.

Mohamed M. K. and Hassan L. M. (1998). Studies on the plant life of the River Nile islands in Minia Governorate. Proc. Sixth Eg. Bot. Conf., Giza, 3 : 481-489.

Raunkiaer C. (1934). The life forms of plants and statistical plant geography. Clarendon Press, Oxford.

Shaheen A. M. (2002 a). Flora of date palm orchards in Egyptian Nubia. Proc. $2^{\text {nd }}$ Int. Conf. Biol. Sci., Tanta Univ., Vol. 2 : 31-45.

Shaheen A. M. (2002 b). Weed diversity of newly farmed land on the southern border of Egypt (eastern and western shores of Lake Nasser).Pakistan Journal of Biological Science, 5: 802-806.

Shaheen A. M., Shedaed M. G., Hamed A. I. and Hamada F. A. (2004). Botanical diversity in the Flora of some islands in the Egyptian Nubia. Proceedings of the First International Conference on Strategy of Egyptian Herbaria Marsh 9-11, Giza, Egypt. pp 162-182.

Stahl R. and Ramadan A. (2008). Environmental Studies on Water Quality of the Ismailia Canal, Egypt. Forschungszentrum Karlsruhe Gmb H, Karlsruhe. pp. 1-58.

Täckholm V. (1974). Students' Flora of Egypt. Second edition Published by Cairo University Printed by Cooperative Printing Company Beirut.

$$
\begin{aligned}
& \text { در اسة على فلورة ترعة الاسماعيلية } \\
& \text { عبدالحليم عبد المجلى محمد - صفوت أمين عازر } \\
& \text { قسم بحوث الفلورة وتصنيف النباتات - معهد بحوث البساتين - مركز البحوث الزر اعية - الجيزة - مصر . }
\end{aligned}
$$

تم تسجيل • (1 انو عا نباتيا فى در اسة لنباتات الفلورة النامية على نزعة الاسماعيلية ، نتبع هذه النباتات ع 9 جنسا

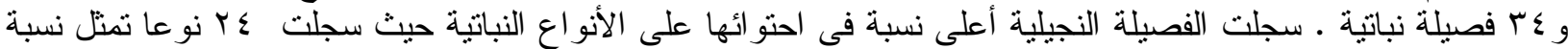

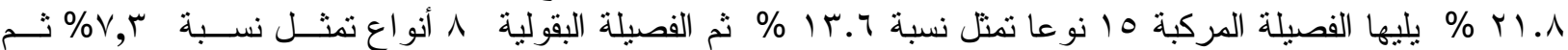

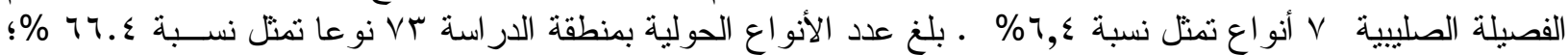

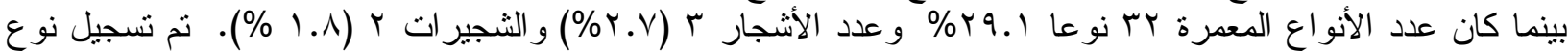

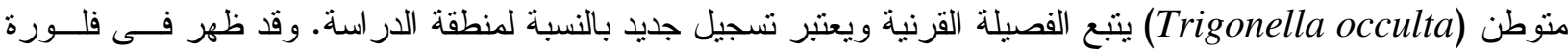

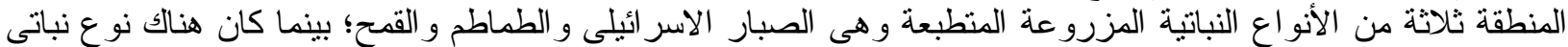

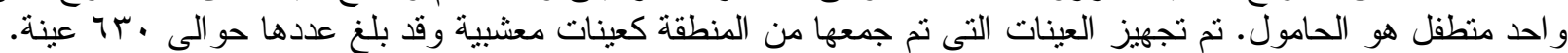

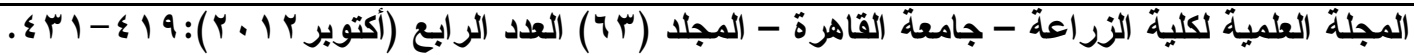

